\title{
Co-occurring internalizing and externalizing psychopathology in childhood and adolescence: a network approach
}

\author{
Eoin McElroy ${ }^{1,2} \cdot$ Mark Shevlin $^{1} \cdot$ Jamie Murphy ${ }^{1} \cdot$ Orla McBride $^{1}$
}

Received: 13 May 2017 / Accepted: 10 February 2018 / Published online: 8 March 2018

(c) The Author(s) 2018. This article is an open access publication

\begin{abstract}
The network approach suggests that psychopathology arises from complex associations between symptoms and may offer insight into the mechanisms that underpin psychiatric comorbidities. The transition from childhood to adolescence is a key period in the development of psychopathology, yet has rarely been considered from a network perspective. As such, the present study examined the network structure of internalizing and externalizing psychopathology from middle childhood through adolescence using data from the Avon Longitudinal Study of Parents and Children (ALSPAC; $n=4405$ ). Eight DSM-IV disorders were assessed using maternal reports when children were aged 7.5, 10.5 and 14 years. Weighted, undirected networks were estimated and the relative importance of each node was assessed using three common measures of node centrality; strength, betweenness, and closeness. A consistent network structure emerged at all three time points; nodes clustered together in two regions of space broadly reflecting the internalizing and externalizing spectra. Permutation tests supported structural invariance across this developmental period. These spectra were bridged by numerous disorder-level interactions, the most consistent of which was between depression and oppositional defiant disorder (ODD). Furthermore, inspection of the centrality indices indicated that generalised anxiety disorder and ODD were the most central disorders in the networks. These findings demonstrate that symptom/disorder-level interplay and reciprocal influence are plausible mechanisms for the association between internalizing and externalizing psychopathology in childhood/adolescence.
\end{abstract}

Keywords Psychopathology $\cdot$ Comorbidity $\cdot$ ALSPAC $\cdot$ Network analysis $\cdot$ Internalizing $\cdot$ Externalizing

\section{Introduction}

Psychiatric comorbidities occur with greater-than-chance frequency [1] and are associated with increased symptom severity and a poorer overall prognosis [2-5]. The mechanisms by which psychiatric symptoms and disorders are related, however, remain poorly understood. Hierarchical dimensional models, which have long been favoured in child and adolescent psychiatric research, account for comorbidity

Electronic supplementary material The online version of this article (https://doi.org/10.1007/s00787-018-1128-x) contains supplementary material, which is available to authorized users.

Eoin McElroy

mcelroy-e1@email.ulster.ac.uk

1 School of Psychology and Psychology Research Institute, Ulster University, Magee Campus, Londonderry, Northern Ireland, UK

2 Department of Psychological Sciences, University of Liverpool, Liverpool, UK by framing psychopathology as a small number of broad transdiagnostic dimensions (e.g. internalizing, externalizing). However, due to strong associations between the dimensions themselves, a general psychopathological factor, $p$, has been proposed to explain the co-occurrence of virtually all psychiatric symptoms and disorders [6, 7]. Although this general factor has been supported in both adult $[6,7]$ and child samples $[8,9]$, a consistent interpretation of the $p$-factor has so far proven elusive [6-11]. The most popular interpretation posits that $p$ reflects a shared aetiological factor, or set of factors (e.g. genetic vulnerability, personality, environmental factors) that predispose individuals to any and all forms of psychopathology [11].

In recent years, an alternative school of thought, the network approach, has gained considerable momentum. This perspective posits that psychiatric disorders reflect complex networks of locally associated symptoms [12]. In such models, variables are presented graphically as nodes (points in space) and the associations between nodes are presented as edges (lines, with thickness denoting strength). This 
approach assumes that the effects of causal factors spread throughout networks via direct, symptom-level interactions and reinforcement (as opposed to causal factors influencing all symptoms simultaneously) [12]. Borsboom and Cramer [12] provide the following chain as an example; chronic stress $\rightarrow$ depressed mood $\rightarrow$ self-reproach $\rightarrow$ insomnia $\rightarrow$ fatigue $\rightarrow$ concentration. As such, the network approach accounts for comorbidity through 'bridging edges'; i.e. direct associations that serve to link reasonably distinct clusters of symptoms/disorders [12,13]. Under such an interpretation, $p$ represents the statistical reduction of a plethora of lower-level interactions between different components of psychopathology. The main advantage of the network approach is that, by focussing on local interactions, we can determine not only how important a symptom/disorder variable is in terms of its overall connectivity (aka centrality), but also where a symptom/disorder is important within the network (i.e. the strongest edges).

Although an abundance of network studies has been published recently, the majority have focussed their enquiry on the structure of single disorders $[14,15]$, or a narrow range of comorbid disorders [16]. To our knowledge, only two studies have used network methods to model broader psychiatric comorbidity; Boschloo and colleagues [17] examined the network structure of 120 symptoms from 12 supposedly distinct DSM-IV disorders in the second wave of the National Epidemiologic Survey on Alcohol and Related Conditions (NESARC, $N=34,653$ ). They found that, although symptoms clustered in a manner broadly consistent with the DSM structure, all 120 symptoms were connected, either directly or indirectly, and each cluster was connected to at least three others. Boschloo et al. [18] then examined the network structure of emotional and behavioural problems in a large sample of pre-adolescents (mean age 11.1 years; $N=2175$ ). Ninety-five symptoms were assessed using the Youth Self-Report [19], and in the resultant network symptoms clustered together in patterns broadly reflective of the YSR domains. Connections were generally stronger and more common within, rather than between, these domains; however, numerous symptom pairs served to link domains, suggesting that the domain boundaries were not as defined as had previously been assumed in factor analytic studies.

Further studies of broad comorbidity networks may help unpack the overlap between higher order dimensions of psychopathology and provide a more complete view of psychiatric comorbidity. The present study expands on previous work in two ways. This is the first study to examine the network structure of comorbid internalizing and externalizing at the disorder level (i.e. where nodes in the network reflect distress/impairment aggregates from internalizing and externalizing symptom groups). To date, network analysis has mainly been used to model the association between symptoms, assuming that symptoms are the base level of psychopathological expression. However, as Borsboom et al. [12] note, symptoms themselves may be broken down into networks of emotional, behavioural and external factors; e.g. difficulty sleeping may be understood in terms of the interplay between mood, melatonin production, routine, caffeine intake and/or screen time. As such, network analysis is flexible and may be used to study psychopathology at various levels of complexity [20]. Indeed, there has been an increase in studies that have examined psychopathology at the construct level [20-23], and this approach has the advantage of reducing the amount of nodes and edges in a network, in turn simplifying interpretation [24]. As the aim of the present study was to provide a network analogue to $p$ (which has primarily been modelled at the disorder-level), we chose to focus our enquiry at the disorder level (e.g. depression, general anxiety, hyperactivity).

Second, this study expands our knowledge of the network structure of psychopathology in childhood and adolescence, and explores whether this structure changes over this key developmental period. The transition from childhood through adolescence is a period marked by significant biological, cognitive and socio-environmental change. It is during this period that psychopathology commonly emerges [1, 25]. Furthermore, disorders that emerge during this period typically serve as precursors to similar problems later in life [26]. As such childhood/adolescence is an ideal period to focus on when examining direct associations between symptoms and/or disorders and may offer key insights into the development of psychiatric sequelae. To date, only one study has focussed on this age group [18]; however, the network structure was examined at only one time point (age 11). The present study examined the network structure of psychopathology (internalizing and externalizing) within a single cohort across three time points $(7.5,10.5$, and 14 years) and tested whether this structure remained stable over this period. Although this study was exploratory in nature, it was predicted that disorders would form two distinct clusters of nodes analogous to the internalizing and externalizing dimensions of psychopathology. No a priori hypotheses were made regarding potential 'bridging edges'.

\section{Method}

\section{Sample}

Data were from the Avon Longitudinal Study of Parents and Children (ALSPAC). The ALSPAC is a prospective cohort study of children born in the English county of Avon between April 1st 1991 and December 31st 1992 $(N=14,062)$. The sample is broadly representative of the overall population of children in the UK [27, 28]. Data were collected using self-report postal questionnaires (completed 
by the study mothers and mother's partners) and via yearly clinics for the study children from the age of 7.5 years [27, 28]. The study website contains details of all the data that are available through a fully searchable data dictionary (http:// www.bris.ac.uk/alspac/researchers/data-access/data-dicti onary/). Ethical approval for the study was obtained from the ALSPAC Ethics and Law Committee and the Local Research Ethics Committees. Further detailed descriptions of the ALSPAC can be found elsewhere [27].

\section{Measures}

Disorders were assessed using maternal report versions of the Development and Wellbeing Assessment (DAWBA) [29]. This structured clinical interview is used to assign psychiatric diagnoses to $5-16$ year olds. It is used to assess fourteen distinct symptom profiles corresponding to ICD-10 and DSM-IV diagnostic criteria. The symptom profiles used in the present analysis were those assessed consistently across the three waves; specific phobia (SPP), social phobia (SOP), posttraumatic stress (PTSD), generalized anxiety (GAD), depression (DEP), attention deficit/hyperactivity disorder (ADHD), oppositional/defiant disorder (ODD) and conduct problems (CD). Official diagnoses were only available at the 7.5 year assessment. To make the use of data from subsequent time points, a comprehensive recoding strategy was employed. Mirroring the structure of the DSM, the DAWBA employs skip patterns; mothers are first asked whether children display core symptoms, followed by questions related to distress and burden associated with symptoms. To create quasi-diagnostic variables that closely mirrored DSM-IV diagnoses, disorders were deemed present if study mothers reported the requisite symptom profiles (including core symptoms) and significant burden or distress associated with these symptom profiles. In the case of ODD, teacher complaint was used in place of distress. For CD, a binary variable reflecting 'any frequent/definite troublesome behaviour' was computed, as per ALSPAC codebook guidelines. This recoding process resulted in eight binary quasi-diagnostic variables at each of the three time points. More detailed descriptions of this recoding process are available in the online supplementary materials.

\section{Missing data}

There is a lack of consensus as to how missing data should best be handled in network analysis [30]. The present study used the most common current practice, listwise deletion $[14,30,31]$. Complete data were available for 4405 maternal reports (DAWBA) at ages 7.5, 10.5 and 14 , and this subsample was used for analysis.

\section{Statistical analysis}

Networks were constructed using the R package 'Isingfit' [32] which was developed to construct weighted undirected networks using binary data. This package uses the elasso method; based on the Ising [33] model, each variable is regressed on all other variables iteratively with a lasso penalty (l1) imposed on the regression coefficients that helps identify the simplest network by balancing sparsity and goodness of fit [18]. The $l 1$ process identifies the best fitting network structure by specifying competing models with different levels of sparsity, and comparing the models using the extended Bayesian information criteria (EBIC) [34]. The edges in these networks are the mean values of the two logistic regression coefficients (i.e. node A predicting node $\mathrm{B}$, and node $\mathrm{B}$ predicting node $\mathrm{A}$ ), which can be interpreted similar to partial correlations. 'Isingfit' was used to construct networks using the 8 binary psychological disorder variables at ages $7.5,10.5$ and 14 years. The resultant networks were then graphically illustrated using the 'qgraph' package [35]. This package uses the Fruchterman-Reingold algorithm to place nodes with stronger and/or more connections closer together [36].

The relative importance of each node to the overall network structure was quantified using three common measures of node centrality. Strength is calculated for each node by summing its weighted connections with other nodes [37]. A node that is high in strength strongly and directly transmits its effects throughout the network [37]. Closeness reflects the average distance from a node of interest to all other nodes in a given network [37]. High closeness means a node is strongly influenced by changes in other nodes in the network [37]. Betweenness is calculated by counting the number of times a node of interest lies on the shortest path between two other nodes [37]. Nodes that are high in betweenness are important for transmitting effects between other nodes in the network. For all measures of centrality, higher values (presented as $z$-scores) are indicative of greater importance to the network as a whole [38].

Recently, the accuracy and stability of networks have received attention in the literature [39]. Accuracy and stability refer to the degree of certainty with which we can interpret the rank ordering the various edge weights and centrality indices. Network accuracy and stability were assessed using the guidelines of Epskamp, Borsboom, and Fried [39]. First, bootstrapped 95\% confidence intervals (CIs) were used to examine the accuracy of network edges. Second, the stability of the order of the centrality indices (strongest to weakest) was examined using a subsetting bootstrap method, i.e. by re-estimating the network based on increasingly smaller subsets of the original sample. The underlying logic of this method is that if the order of centrality estimates from a network based on a small subset is highly correlated 
to the order of the centrality from the original network, the centrality estimates can be considered stable [39]. Stability analyses were conducted using the R package 'bootnet' [39].

To test for changes in the relationships between internalizing and externalizing disorders over time, structural invariance was examined using the 'Network Comparison Test' (NCT) package in R [40]. NCT tests the null hypothesis $A_{1}=A_{2}$, where $A_{1}$ and $A_{2}$ are matrices containing the strengths of connections in two separate networks [40]. The NCT procedure involves non-parametric permutation testing and is conducted in three phases [40]. First the two networks in question are estimated and the maximum difference in edge strength between two given networks $(M)$ is calculated and serves as the test statistic [40]. For the second step, cases are repeatedly randomly swapped between groups and the networks and test statistics re-estimated. Third, a reference distribution is created from these test statistics and statistical significance is determined, with the $p$ value equal to the proportion of test statistics that have an equal or higher value than the observed test statistic [40].

\section{Results}

\section{Descriptive statistics}

Table 1 shows the frequencies and relative percentages of the disorders at the different time points. Across time, GAD was the most common disorder, followed by SPP. PTSD was the least endorsed disorder. Bivariate correlations between disorders are presented in the online supplementary materials (Table S1).

\section{Association networks}

The association networks, constructed separately at each time point, are presented in Fig. 1. Similar patterns of association were observed; two distinct clusters of nodes emerged, reflecting internalizing and externalizing disorders. Within the internalizing cluster, GAD was placed centrally and demonstrated moderate to strong associations with all other disorders. Within the externalizing cluster, thick edges indicated strong associations between ODD and ADHD at all three time points. Relationships between ADHD and CD, however, were comparatively weak. Indeed, ODD appeared to bridge the associations between $\mathrm{CD}$ and $\mathrm{ADHD}$ at the different time points.

GAD appeared to lie at the heart of the network as a whole, due to its central placing at all three time points. An inspection of the centrality indices (Fig. 2) corroborated this observation; across time GAD consistently scored highest on the measures of strength, closeness and betweenness. Within the externalizing cluster, ODD was the most central node
Table 1 Frequencies and relative percentages of measured indicators by assessment period

\begin{tabular}{llll}
\hline & 7.5 years & 10.5 years & 14 years \\
\hline SPP & & & \\
Present & $209(4.7 \%)$ & $635(14 \%)$ & $446(10.1 \%)$ \\
Absent & 4196 & 3770 & 3959 \\
SOP & & & \\
Present & $162(3.6 \%)$ & $197(4.5 \%)$ & $236(5.4 \%)$ \\
Absent & 4243 & 4208 & 4169 \\
PTSD & & & $134(3 \%)$ \\
Present & $63(1.4 \%)$ & $100(2.3 \%)$ & 4271 \\
Absent & 4342 & 4305 & $541(12.3 \%)$ \\
GAD & & & 3864 \\
Present & $222(5 \%)$ & $514(11.7 \%)$ & \\
Absent & 4183 & 3891 & $464(10.5 \%)$ \\
DEP & & & 3941 \\
Present & $201(4.5 \%)$ & $412(9.4 \%)$ & 4175 \\
Absent & 4202 & 3993 & $3964(10 \%)$ \\
ADHD & & & $245(5.6 \%)$ \\
Present & $230(5.5 \%)$ & $340(7.7 \%)$ & \\
Absent & 4175 & 4065 & \\
ODD & & $184(4.2)$ & \\
Present & $163(3.7 \%)$ & $190(4.3 \%)$ & \\
Absent & 4242 & 4215 & \\
CD & & & \\
Present & $256(5.8 \%)$ & 4221 & \\
Absent & 4242 & & \\
\hline
\end{tabular}

$S P P$ specific phobia, $S O P$ social phobia, $P T S D$ post-traumatic stress disorder, $G A D$ generalized anxiety disorder, $D E P$ major depression, $A D H D$ attention deficit hyperactivity disorder, $O D D$ oppositional/ defiant disorder, $C D$ conduct disorder

at ages 10.5 and 14 years, whereas ADHD demonstrated greater betweenness and closeness at age 7.5 years. Crosscluster associations were common, although generally of smaller magnitude compared to the within-cluster associations. The most consistent bridging edge was DEP-ODD, which consistently appeared within the 10 strongest edges (Fig S1). The edge GAD-ADHD was also significant at each time point. Other edges were less consistent. For example, the edge SOP-ADHD was moderately strong at ages 10.5 and 14 years, but non-significant at age 7.5.

The bootstrapped $95 \%$ confidence intervals for the edges are presented in the online supplementary materials (Fig S1). Although there was considerable overlap among the CIs, the strongest edges demonstrated little overlap, suggesting statistically significant differences in the strengths of these associations. As such, the networks were moderately accurately estimated, and the order of the edge weights in each of the three networks can be interpreted with some degree of confidence [48]. The results from the subsetting bootstrap method are presented in the online supplementary 
Age $7.5(n=4,405)$

Age $10.5(n=4,405)$

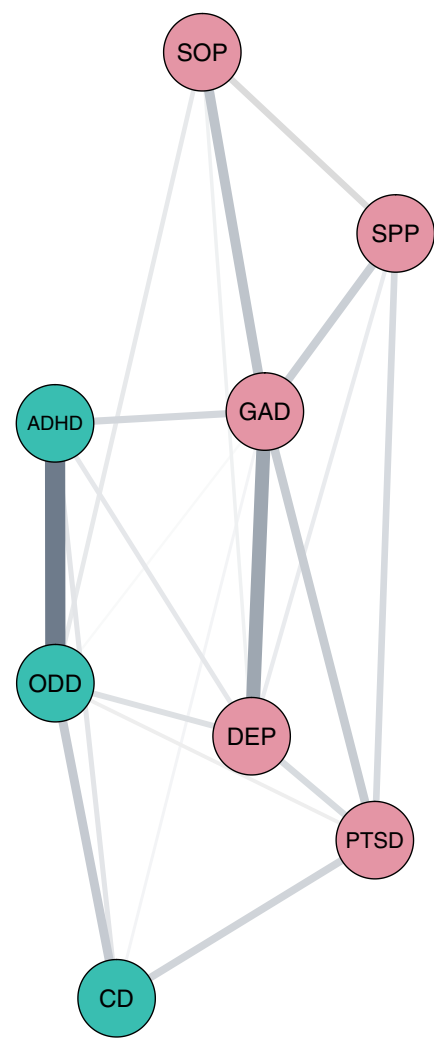

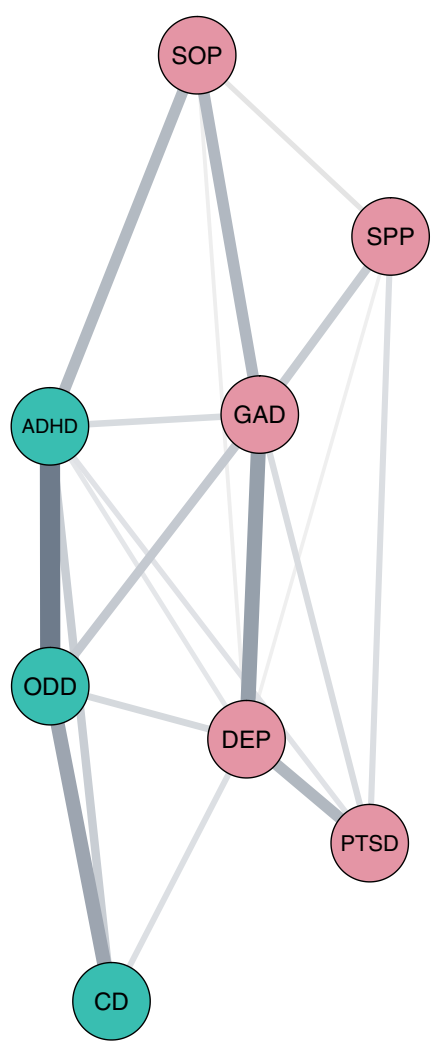

Age $14(n=4,405)$

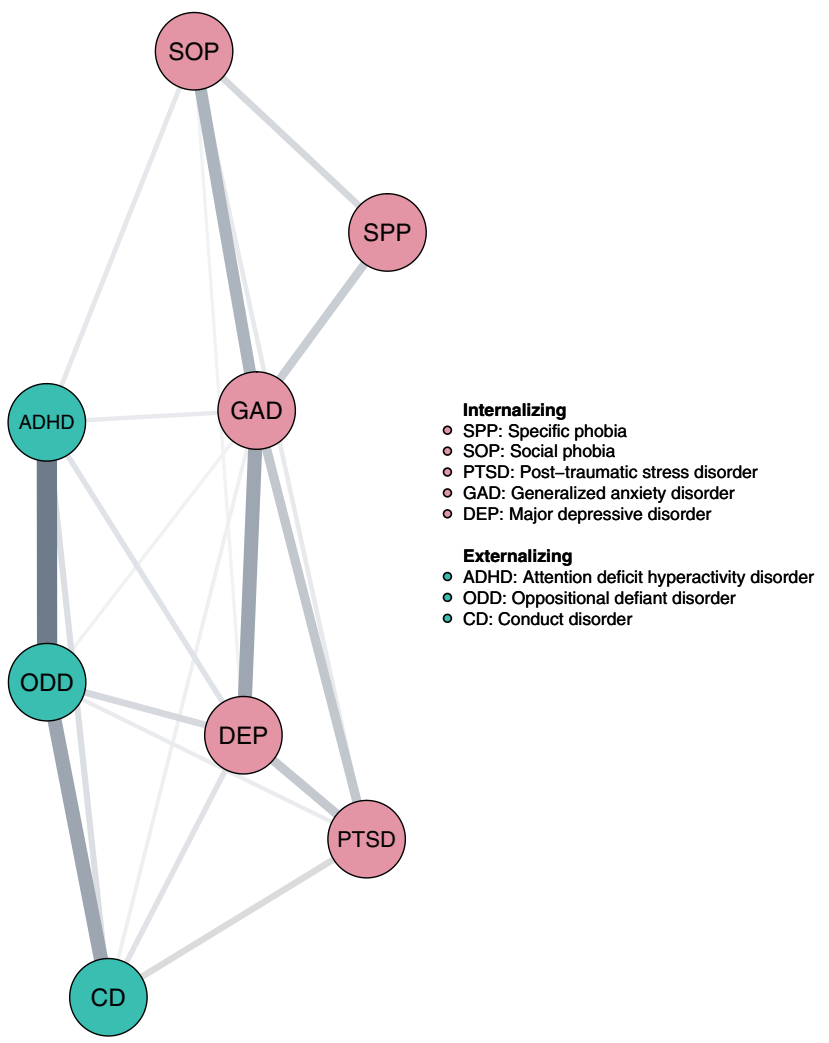

Fig. 1 Association networks at ages 7.5, 10.5, and 14 years. Line thickness denotes strength of association. All edges are positive

materials (Fig S2). Overall, the network centrality indices appeared highly stable; the correlations between the measures of centrality using the full sample and a subset of $30 \%$ ranged from approximately $0.5-0.85$. This indicates that, even when $70 \%$ of the sample were randomly removed, the order of the centrality indices remained stable, suggesting a robust estimation of centrality. Overall, strength was the most stable of the three indices, and betweenness the weakest.

With regard to structural invariance, after applying a Bonferroni adjustment to account for multiple testing, the nonparametric permutation tests found no significant difference in overall network structure (Fig S3). As such, the network structure was considered broadly stable over time.

\section{Discussion}

The present study sought to examine the network structure of internalizing and externalizing disorders assessed between middle childhood and early adolescence. To our knowledge, this is the first study to take a network approach to the modelling of internalizing and externalizing at the disorder level. Furthermore, this is the first study to apply network techniques to a cohort of children assessed from middle childhood through adolescence. A coherent network structure was identified at three time points (age 7.5, 10.5 and 14 years), and tests of structural invariance indicated that the networks remained generally stable, despite the many biological, cognitive and social changes that typically occur over this developmental period. As predicted, two regions of clustered nodes emerged, reflecting strong associations between internalizing disorders, and externalizing disorders. These clusters were most strongly bridged via the edges GAD-ADHD, and DEP-ODD. The centrality indices indicated that GAD and ODD were most important to the networks as a whole. An examination of the robustness of the centrality indices suggested that they could be interpreted with a degree of confidence.

\section{Internalizing and externalizing as networks}

Borsboom and colleagues [41] suggested that, if modelled using network techniques, traditional hierarchical measurement models of psychopathology would be reflected in clusters of highly associated nodes, analogous to the higher order dimensions of internalizing and externalizing. The findings of the present study support this claim. While 
Fig. 2 Centrality statistics for association networks at the three time points. $S P P$ specific phobia, $S O P$ social phobia, $P T S D$ post-traumatic stress disorder, $G A D$ generalized anxiety disorder, $D E P$ major depression, $A D H D$ attention deficit hyperactivity disorder, $O D D$ oppositional defiant disorder, $C D$ conduct disorder. Centrality values ( $y$ axis) presented as $z$-scores

\section{Strength}

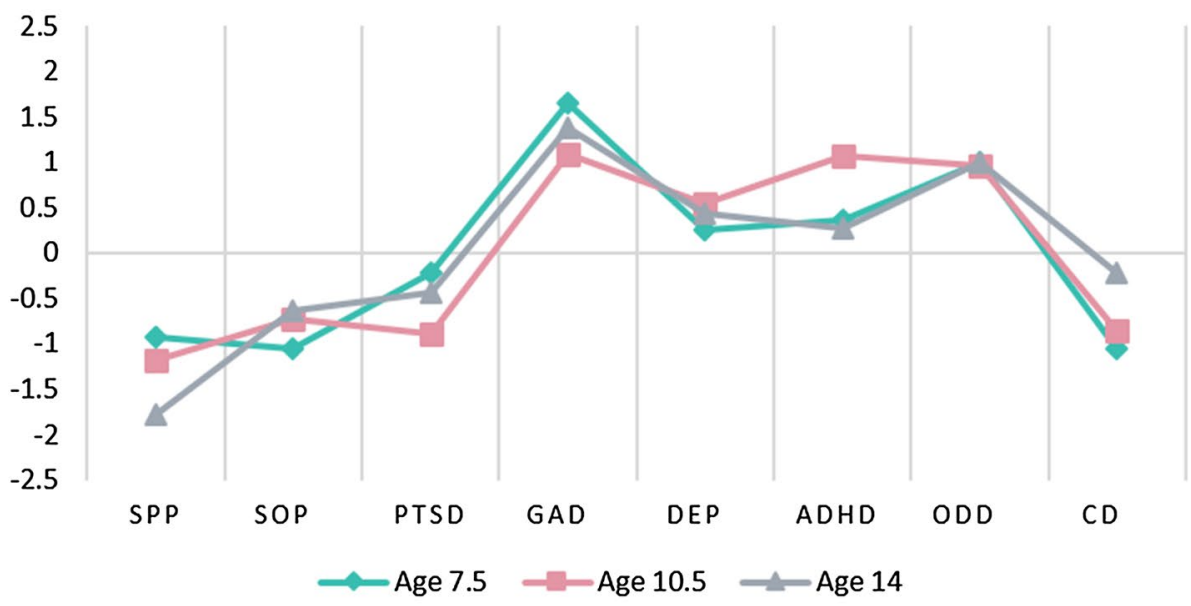

\section{Closeness}

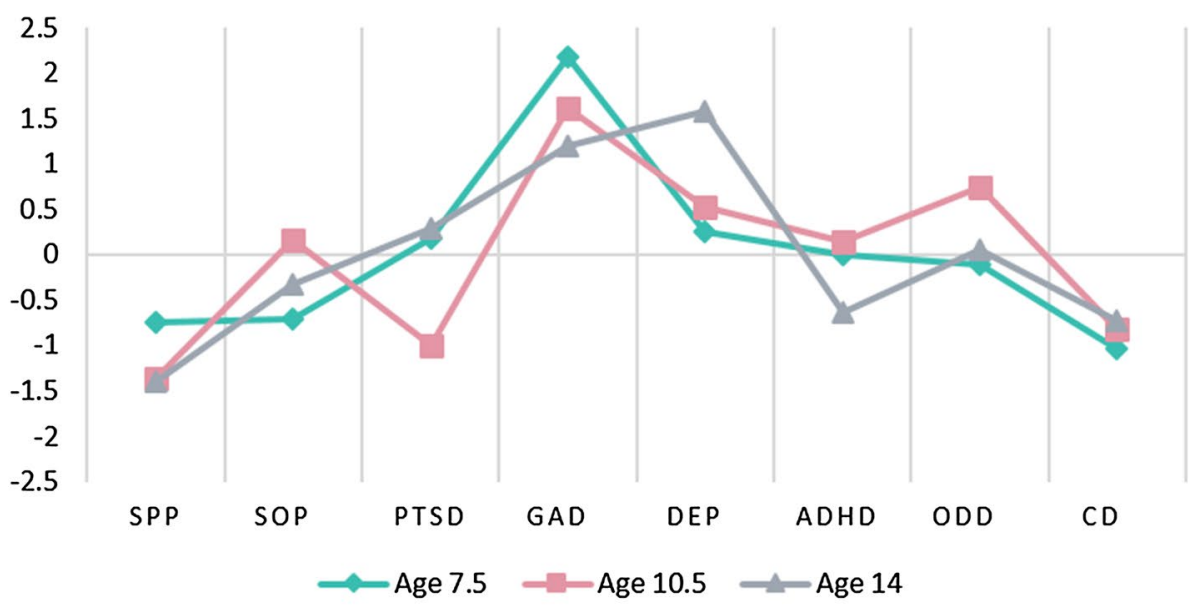

\section{Betweenness}

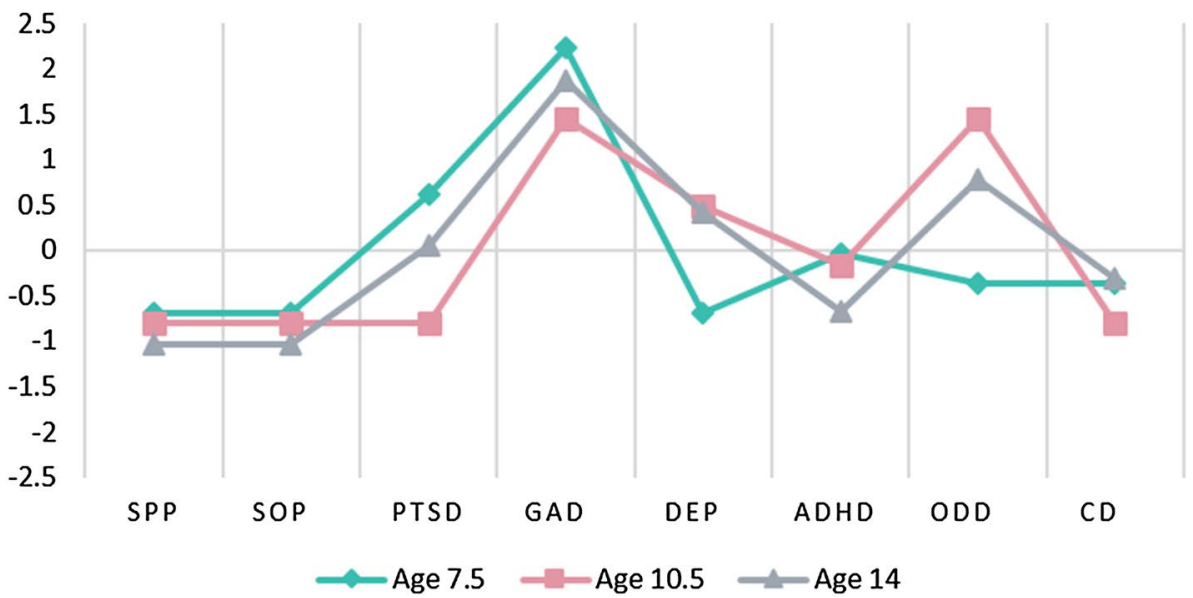


this finding may not be surprising (both network and latent variable models are derived from covariance), it offers a different interpretation of psychiatric comorbidity. This perspective suggests that, rather than a single causal factor (or amalgam of causal factors) driving the association between symptoms/disorders, causal factors spread their effects throughout psychopathological networks via local interactions and reinforcement. In other words, higher order dimensions (e.g. $p$ ) may be capturing a plethora of locallevel interactions. Under this interpretation, the dimensions of internalizing and externalizing are correlated due to certain disorders (which themselves are comprised of networks of associated symptoms) acting as 'bridges' between these two broad spectra.

The nature of these interactions (i.e. edges), however, is far from clear and serves to further emphasise the complexity of psychiatric comorbidity. Given that the analysed data were cross-sectional (within-time points), a significant edge could represent a multitude of possible relationships. First, it is possible that disorders directly influence each other. For example, there is a long history of research looking at the comorbid anxiety and depression, with evidence suggesting that anxiety tends to precede and lead to subsequent depression [42]. One proposed mechanism for such a relationship is that cognitive/neurophysiological processes (e.g. sustained heightened physiological arousal) may lead to an exhaustion of the body which manifests as depression [43]. Other edges may reflect more indirect associations, e.g. the edges ODDDEP and ADHD-GAD in the current networks. Developmental cascade models have long suggested that externalizing behaviour may indirectly lead to internalizing problems through mediating variables [44-46]. To illustrate, frequent disruptive behaviour in childhood/adolescence may lead to negative reactions from parents, teachers and/or peers, e.g. shouting, punishment, ostracisation from peer group, and/ or academic failure. Such negative outcomes may in turn foster feelings of irritability, distress and worthlessness within the child, and if left unchecked, these experiences may eventually progress to levels of clinical significance $[45,46]$. Edges in any given network may also represent spurious associations due to an unmeasured common cause. For example, shared biological (e.g. genes) or environmental (e.g. trauma) risk factors may influence the development of multiple symptom domains simultaneously [42]. If these risk factors are not represented in a given network, this may give rise to non-causal associations between disorders. Edges are further complicated by the possibility of bidirectional feedback loops, equifinality (multiple risk factors, pathways and processes leading to similar outcomes), and multifinality (specific risk factors leading to multiple outcomes) [47].

Given that the networks in the present study were undirected and cross-sectional, any causal interpretations, such as those described above, are purely speculative. The aim of the present study, however, was not to infer strict causal relationships but to demonstrate how network analysis can be used to quantify the importance of disorders and identify key associations between disorder pairs and as such generate hypotheses regarding the complex mechanisms that drive psychiatric comorbidity. For example, in the present study, GAD and ODD were identified as the two most influential nodes within wider comorbidity networks, suggesting that symptoms within these disorders may be influential in the initiation and/or maintenance of comorbid psychopathology. Furthermore, a number of significant edges bridged the spectra of internalizing and externalizing, the strongest of which was the edge ODD-DEP. This suggests that local-level interactions between these two disorders may go some way to explaining the correlations between internalizing and externalizing when modelled as continuous dimensions [24].

\section{Strengths and limitations of the present study}

The main strength of the present study was the large sample size relative to the number of parameters estimated [49]. With regard to limitations, diagnostic data were not available for the participants at all three of the time points; therefore, a comprehensive recoding strategy was adopted. It must be noted, however, that the diagnostic algorithms used to create the quasi-diagnostic variables were based on skip patterns (i.e. those who did not endorse 'core' symptoms were scored as having no disorder, and subsequent distress and burden were not assessed). This likely introduces an element of bias to the data in favour of DSM scoring conventions. Similar bias likely affects all analyses that employ DSM skip structures.

Skip patterns are particularly problematic when using symptom-level data, as they introduce deterministic dependencies (i.e. secondary symptoms can only be endorsed if primary symptoms are first endorsed, thus artificially inflating correlations between these symptom pairs). However, this was not the case in the present study, as a diagnosis of one disorder was not dependent on the diagnosis of another disorder. Finally, any study that employs network analysis comes with the caveat that this approach is highly divisive. Indeed, this has been evidenced by a series of back-and-forth papers focussed on the replicability of networks [50-52]. A methodological critique of network analysis and/or traditional latent variable models is beyond the scope of this article (for recent reviews, see [13, 48, 50-52]); however, our findings do appear to support the replicability of psychopathological networks within a cohort of children and adolescents assessed repeatedly over a period of significant development. 


\section{Conclusion}

In conclusion, the present study sought to model internalizing and externalizing disorders in childhood and adolescence as a series of networks. A consistent and relatively stable network structure emerged across time, in which nodes formed two clusters broadly reflecting internalizing and externalizing. The two domains appeared to be linked through numerous bridging edges, and generalized anxiety disorder (GAD) and oppositional defiant disorder (ODD) were the most influential nodes in the networks. These findings offer a plausible interpretation of the $p$-factor model of comorbidity; i.e. the correlations between internalizing and externalizing dimensions may be due to direct associations between symptoms and/or disorders.

Acknowledgements We are extremely grateful to all the families who took part in this study, the midwives for their help in recruiting them, and the whole ALSPAC team, which includes interviewers, computer and laboratory technicians, clerical workers, research scientists, volunteers, managers, receptionists and nurses. The UK Medical Research Council and the Wellcome Trust (Grant ref: 092731) and the University of Bristol provide core support for ALSPAC. This publication is the work of the authors and the corresponding author will serve as guarantor for the contents of this paper.

\section{Compliance with ethical standards}

Conflict of interest On behalf of all authors, the corresponding author states that there is no conflict of interest.

Open Access This article is distributed under the terms of the Creative Commons Attribution 4.0 International License (http://creativeco mmons.org/licenses/by/4.0/), which permits unrestricted use, distribution, and reproduction in any medium, provided you give appropriate credit to the original author(s) and the source, provide a link to the Creative Commons license, and indicate if changes were made.

\section{References}

1. Kessler RC, Berglund P, Demler O, Jin R, Merikangas KR, Walters EE (2005) Lifetime prevalence and age-of-onset distributions of DSM-IV disorders in the National Comorbidity Survey Replication. Arch Gen Psychiatry 62:593-602. https:// doi.org/10.1001/archpsyc.62.6.593

2. McElroy E, Shevlin M, Murphy J (2017) Internalizing and externalizing disorders in childhood and adolescence: a latent transition analysis using ALSPAC data. Compr Psychiatry 75:75-84. https://doi.org/10.1016/j.comppsych.2017.03.003

3. Nock MK, Hwang I, Sampson NA, Kessler RC (2010) Mental disorders, comorbidity and suicidal behavior: results from the National Comorbidity Survey Replication. Molecular Psychiatry 15:868-876. https://doi.org/10.1038/mp.2009.29

4. Angold A, Costello EJ, Erkanli A (1999) Comorbidity. J Child Psychol Psychiatry 40:57-87. https://doi. org/10.1111/1469-7610.00424
5. Angst J, Sellaro R, Merikangas KR (2002) Multimorbidity of psychiatric disorders as an indicator of clinical severity. Eur Arch Psychiatry Clin Neurosci 252:147-154. https://doi. org/10.1007/s00406-002-0357-6

6. Caspi A, Houts RM, Belsky DW, Goldman-Mellor SJ, Harrington $\mathrm{H}$, Israel S et al (2014) The $p$ factor: one general psychopathology factor in the structure of psychiatric disorders? Clin Psychol Sci 2:119-137. https://doi.org/10.1177/21677 02613497473

7. Lahey BB, Applegate B, Hakes JK, Zald DH, Hariri AR, Rathouz PJ (2012) Is there a general factor of prevalent psychopathology during adulthood? J Abnorm Psychol 121:971. https://doi. org/10.1037/a0028355

8. Patalay P, Fonagy P, Deighton J, Belsky J, Vostanis P, Wolpert M (2015) A general psychopathology factor in early adolescence. $\mathrm{Br}$ J Psychiatry 207:15-22. https://doi.org/10.1192/bjp.bp.114.14959 1

9. Martel MM, Pan PM, Hoffmann MS, Gadelha A, do Rosário MC, Mari JJ et al (2017) A general psychopathology factor (P factor) in children: structural model analysis and external validation through familial risk and child global executive function. J Abnorm Psychol 126:137-148. https://doi.org/10.1037/abn0000205

10. Greene AL, Eaton NR (2017) The temporal stability of the bifactor model of comorbidity: an examination of moderated continuity pathways. Compr Psychiatry 72:74-82. https://doi.org/10.1016/j. comppsych.2016.09.010

11. Murray AL, Eisner M, Ribeaud D (2016) The development of the general factor of psychopathology ' $p$ factor'through childhood and adolescence. J Abnorm Child Psychol 44:1573-1586. https://doi. org/10.1007/s10802-016-0132-1

12. Borsboom D, Cramer AO (2013) Network analysis: an integrative approach to the structure of psychopathology. Ann Rev Clin Psychol 9:91-121. https://doi.org/10.1146/annurev-clinpsy-05021 2-185608

13. Fried EI, van Borkulo CD, Cramer AO, Boschloo L, Schoevers RA, Borsboom D (2017) Mental disorders as networks of problems: a review of recent insights. Soc Psychiatry Psychiatr Epidemiol 52:1-10. https://doi.org/10.1007/s00127-016-1319-z

14. Fried EI, Epskamp S, Nesse RM, Tuerlinckx F, Borsboom D (2016) What are'good'depression symptoms? Comparing the centrality of DSM and non-DSM symptoms of depression in a network analysis. J Affect Disord 189:314-320. https://doi. org/10.1016/j.jad.2015.09.005

15. McNally RJ, Robinaugh DJ, Wu GW, Wang L, Deserno MK, Borsboom D (2015) Mental disorders as causal systems: a network approach to posttraumatic stress disorder. Clin Psychol Sci 3:836-849. https://doi.org/10.1177/2167702614553230

16. Beard C, Millner A, Forgeard M, Fried E, Hsu K, Treadway M et al (2016) Network analysis of depression and anxiety symptom relationships in a psychiatric sample. Psychol Med 46:3359-3369. https://doi.org/10.1017/S0033291716002300

17. Boschloo L, van Borkulo CD, Rhemtulla M, Keyes KM, Borsboom D, Schoevers RA (2015) The network structure of symptoms of the diagnostic and statistical manual of mental disorders. PLoS One 10:e0137621. https://doi.org/10.1371/journ al.pone. 0137621

18. Boschloo L, Schoevers RA, van Borkulo CD, Borsboom D, Oldehinkel AJ (2016) The network structure of psychopathology in a community sample of preadolescents. J Abnorm Psychol 125:599. https://doi.org/10.1037/abn0000150

19. Achenbach TM (1991) Manual for the youth self-report and 1991 profile. Department of Psychiatry, University of Vermont, Burlington

20. Anker JJ, Forbes MK, Almquist ZW, Menk JS, Thuras P, Unruh AS, Kushner MG (2017) A network approach to modeling 
comorbid internalizing and alcohol use disorders. J Abnorm Psychol 126:325-339. https://doi.org/10.1037/abn0000257

21. Hoorelbeke K, Marchetti I, De Schryver M, Koster EH (2016) The interplay between cognitive risk and resilience factors in remitted depression: a network analysis. J Affect Disord 195:96-104. https ://doi.org/10.1016/j.jad.2016.02.001

22. Isvoranu A-M, Borsboom D, van Os J, Guloksuz S (2016) A network approach to environmental impact in psychotic disorder: brief theoretical framework. Schizophr Bull 42:870-873. https:// doi.org/10.1093/schbul/sbw049

23. Costantini G, Richetin J, Borsboom D, Fried EI, Rhemtulla M, Perugini M (2015) Development of indirect measures of conscientiousness: combining a facets approach and network analysis. Eur J Pers 29:548-567. https://doi.org/10.1002/per.2014

24. Krueger RF, DeYoung CG, Markon KE (2010) Toward scientifically useful quantitative models of psychopathology: the importance of a comparative approach. Behav Brain Sci 33:163-164. https://doi.org/10.1017/S0140525X10000646

25. Paus T, Giedd JN, Keshavan M (2008) Why do many psychiatric disorders emerge during adolescence? Nat Rev Neurosci 9:947957. https://doi.org/10.1038/nrn2513

26. Copeland WE, Shanahan L, Costello EJ, Angold A (2009) Childhood and adolescent psychiatric disorders as predictors of young adult disorders. Arch Gen Psychiatry 66:764-772. https://doi. org/10.1001/archgenpsychiatry.2009.85

27. Boyd A, Golding J, Macleod J, Lawlor DA, Fraser A, Henderson J et al (2013) Cohort profile: the 'children of the $90 \mathrm{~s}$ '— the index offspring of the Avon Longitudinal Study of Parents and Children. Int J Epidemiol 42:111-127. https://doi.org/10.1093/ije/dys064

28. Fraser A, Macdonald-Wallis C, Tilling K, Boyd A, Golding J, Davey Smith G et al (2012) Cohort profile: the Avon Longitudinal Study of Parents and Children: ALSPAC mothers cohort. Int J Epidemiol 42:97-110. https://doi.org/10.1093/ije/dys066

29. Goodman R, Ford T, Richards H, Gatward R, Meltzer H (2000) The development and well-being assessment: description and initial validation of an integrated assessment of child and adolescent psychopathology. J Child Psychol Psychiatry Allied Discipl 41:645-655. https://doi.org/10.1111/j.1469-7610.2000.tb02345.x

30. Santos H, Fried EI, Asafu-Adjei J, Ruiz RJ (2017) Network structure of perinatal depressive symptoms in Latinas: relationship to stress and reproductive biomarkers. Res Nurs Health 40:218-228. https://doi.org/10.1002/nur.21784

31. Isvoranu A-M, van Borkulo CD, Boyette L-L, Wigman JT, Vinkers CH, Borsboom D et al (2016) A network approach to psychosis: pathways between childhood trauma and psychotic symptoms. Schizophr Bull 43:187-196. https://doi.org/10.1093/schbul/sbw05 5

32. van Borkulo C, Epskamp S (2014) IsingFit: Fitting Ising models using the eLasso method. $\mathrm{R}$ package version 020

33. Ising E (1925) Beitrag zur theorie des ferromagnetismus. Zeitschrift für Physik 31:253-258

34. Chen J, Chen Z (2008) Extended Bayesian information criteria for model selection with large model spaces. Biometrika 95:759-771. https://doi.org/10.1093/biomet/asn034

35. Epskamp S, Cramer AO, Waldorp LJ, Schmittmann VD, Borsboom D (2012) qgraph: network visualizations of relationships in psychometric data. J Stat Soft. 48:1-18. https://doi.org/10.18637/ jss.v048.i04

36. Fruchterman TM, Reingold EM (1991) Graph drawing by force-directed placement. Software 21:1129-1164. https://doi. org/10.1002/spe.4380211102
37. Costantini G, Epskamp S, Borsboom D, Perugini M, Mõttus R, Waldorp LJ et al (2015) State of the aRt personality research: a tutorial on network analysis of personality data in R. J Res Pers 54:13-29. https://doi.org/10.1016/j.jrp.2014.07.003

38. Opsahl T, Agneessens F, Skvoretz J (2010) Node centrality in weighted networks: generalizing degree and shortest paths. Social Networks 32:245-251. https://doi.org/10.1016/j.socne t.2010.03.006

39. Epskamp S, Borsboom D, Fried EI (2017) Estimating psychological networks and their accuracy: a tutorial paper. Behav Res Methods. https://doi.org/10.3758/s13428-017-0862-1

40. van Borkulo C, Boschloo L, Kossakowski J, Tio P, Schoevers R, Borsboom D, et al. (Manuscript submitted for publication) Comparing network structures on three aspects: a permutation test

41. Borsboom D, Cramer AO, Schmittmann VD, Epskamp S, Waldorp LJ (2011) The small world of psychopathology. PLoS One 6:e27407. https://doi.org/10.1371/journal.pone.0027407

42. Cummings CM, Caporino NE, Kendall PC (2014) Comorbidity of anxiety and depression in children and adolescents: 20 years after. Psychol Bull 140:816-845. https://doi.org/10.1037/a0034733

43. Zahn-Waxler C, Klimes-Dougan B, Slattery MJ (2000) Internalizing problems of childhood and adolescence: prospects, pitfalls, and progress in understanding the development of anxiety and depression. Dev Psychopathol 12:443-466

44. Masten AS, Cicchetti D (2010) Developmental cascades. Dev Psychopathol 22:491-495. https://doi.org/10.1017/S095457941 0000222

45. Masten AS, Roisman GI, Long JD, Burt KB, Obradović J, Riley JR et al (2005) Developmental cascades: linking academic achievement and externalizing and internalizing symptoms over 20 years. Dev Psychol 41:733-746. https://doi. org/10.1037/0012-1649.41.5.733

46. Capaldi DM (1992) Co-occurrence of conduct problems and depressive symptoms in early adolescent boys: II. A 2-year follow-up at Grade 8. Dev Psychopathol 4:125-144. https://doi. org/10.1017/S0954579400005605

47. Cicchetti D (2016) Developmental psychopathology, theory and method. Wiley, Hoboken

48. Fried EI, Cramer AO (2017) Moving forward: challenges and directions for psychopathological network theory and methodology. Perspect Psychol Sci 12:999-1020. https://doi. org/10.1177/1745691617705892

49. Epskamp S, Fried EI (2016) A primer on estimating regularized psychological networks. arXiv preprint arXiv:160701367

50. Forbes MK, Wright AG, Markon K, Krueger R (2017) Evidence that psychopathology symptom networks have limited replicability. J Abnorm Psychol 126:969-988. https://doi.org/10.1037/ abn0000276

51. Borsboom D, Fried EI, Epskamp S, Waldorp LJ, van Borkulo CD, van der Maas HL et al (2017) False alarm? A comprehensive reanalysis of "Evidence that psychopathology symptom networks have limited replicability" by Forbes, Wright, Markon, and Krueger. J Abnorm Psychol 126:989-999. https://doi.org/10.1037/ abn0000306

52. Forbes MK, Wright AG, Markon KE, Krueger RF et al (2017) Further evidence that psychopathology networks have limited replicability and utility: response to Borsboom et al. (2017) and Steinley et al. (2017). J Abnorm Psychol 126:1011-1016. https:// doi.org/10.1037/abn0000313 